\title{
Onde estão sendo publicados os estudos sobre suicídio no Brasil?
}

Where are being published the studies about suicide in Brazil?

\section{Sr. Editor,}

Recentemente, ocorreu em nossa cidade, Belo Horizonte, o II Congresso da Associação de Suicidologia da América Latina e Caribe, evento que reuniu aproximadamente 350 participantes e que se destacou pela excelente qualidade dos trabalhos científicos nacionais e internacionais apresentados durante seus três dias de duração.

Fato que chamou a atenção foi a grande quantidade de trabaIhos realizados por grupos de pesquisa nacionais, abordando o tema em praticamente todos os aspectos possíveis, desde uma visão antropológica, passando pela psicanálise e chegando a avanços neurobiológicos.

A partir disso, tendo em vista que o suicídio é considerado uma epidemia no âmbito da saúde ${ }^{1}$ e que se torna de fundamental importância a divulgação dos dados obtidos pelos diversos grupos de pesquisa, resolvemos averiguar a produção científica a respeito da suicidologia nas três principais revistas científicas psiquiátricas brasileiras: Revista Brasileira de Psiquiatria 
(RBP), Revista de Psiquiatria Clínica e Revista de Psiquiatria do Rio Grande do Sul.

Os periódicos foram acessados pela The Scientific Electronic Library Online (SCIELO), onde todos os exemplares disponíveis foram avaliados. (RBP $=34$ exemplares; Revista de Psiquiatria Clínica $=26$ exemplares; e Revista de Psiquiatria do Rio Grande do Sul $=11$ exemplares). Como cada periódico tem formato diferente e publica artigos com diversas características, como editoriais, revisões, cartas, artigos completos, entre outros, consideramos, em nossa análise, todos os tipos de artigo, excetuando-se apenas comentários sobre livros e erratas. Não incluímos em nosso estudo suplementos de nenhuma das revistas. Assim, a partir dos exemplares, avaliamos todos os artigos publicados nos periódicos visando achar aqueles cujo tema primário fosse a suicidologia.

No total, obtivemos 844 artigos, com predomínio da Revista Brasileira de Psiquiatria $(64,45 \%)(p<0,05)$. Obtivemos 15 artigos cuja temática principal foi o suicídio, correspondendo a $1,77 \%$ do total de publicações. Novamente, a RBP apresentou maior representatividade, com 11 artigos publicados (73,33\%) $(p<0,05)$ - Tabela 1

Tabela 1 - Artigos sobre suicidologia publicados nas três principais revistas de psiquiatria no Brasil

\begin{tabular}{lcc}
\hline \multicolumn{1}{c}{ Periódico } & $\begin{array}{c}\text { Número total } \\
\text { de artigos }\end{array}$ & $\begin{array}{c}\text { Número de artigos } \\
\text { sobre suicidologia }\end{array}$ \\
\hline $\begin{array}{l}\text { RBP } \\
\text { Revista de Psiquiatria } \\
\text { Clinica }\end{array}$ & $544(64.45 \%)$ & $11(73.33 \%)$ \\
$\begin{array}{l}\text { Revista de Psiquiatria do } \\
\text { Rio Grande do Sul }\end{array}$ & $164(19.43 \%)$ & $1(6.66 \%)$ \\
\hline Total & $136(16.11 \%)$ & $3(20.00 \%)$ \\
\hline
\end{tabular}

*Proporção do número total de artigos: RBP x Revista de Psiquiatria Clínica: $p=\langle 0,05$; RBP $\times$ Revista de Psiquiatria do Rio Grande do Sul: $\boldsymbol{p}<0,05$; Revista de Psiquiatria Clínica x Revista de Psiquiatria do Rio Grande do Sul: $p>0,05$.

"*Proporção de número de artigos sobre suicidologia: RBP $x$ Revista de Psiquiatria Clinica: $\mathbf{p}=<0,05 ; R B P \times$ Revista de Psiquiatria do Rio Grande do Sul: $\boldsymbol{p}<0,05$; Revista de Psiquiatria Clínica $\times$ Revista de Psiquiatria do Rio Grande do Sul: $\boldsymbol{p}<0,05$.

OBS: Foram utilizados os teste qui-quadrado e exato de Fisher

Várias dúvidas e questionamentos podem ser tirados desses dados que, apesar de simples, são de fundamental importância.

Primeiro: onde estão publicados os achados sobre o tema? Claro que, sendo o suicídio um tema muito abrangente, diversos outros tipos de publicações podem estar abrigando tais resultados como, por exemplo, periódicos sobre saúde pública. Apesar dessa ressalva, o número de publicações a respeito do tema chama a atenção pelo seu pequeno número, já que se trata de assunto de extrema relevância. ${ }^{1}$

Segundo: se os estudos estão sendo publicados em periódicos internacionais, por que não priorizar veículos nacionais, facilitando a disseminação dos dados entre instituições e serviços de saúde brasileiros? Além disso, recentemente a RBP foi indexada pelo ISI, refletindo sua qualidade em âmbito internacional, além do fato de as outras duas revistas serem indexadas pela SCIELO, veículo também revelador da qualidade científica dessas publicações. ${ }^{2-4}$

Terceiro: por último, se esses dados não estão sendo publicados, como poderemos efetivar políticas de saúde eficientes em nosso país se não compreendemos o fenômeno do suicídio como um todo? ${ }^{5}$

Como foi enfatizado no evento, no que se trata a respeito do suicídio, é preciso "Compreender para Prevenir"... Mas, para podermos compreender, precisamos divulgar.
Felipe Filardi da Rocha, Humberto Corrêa Serviço de Psiquiatria, Hospital das Clínicas, Universidade Federal de Minas Gerais (UFMG), Belo Horizonte (MG), Brasil Departamento de Farmacologia, Instituto de Ciências Biológicas, Universidade Federal de Minas Gerais (UFMG), Belo Horizonte (MG), Brasil

Naira Vassalo Lage

Departamento de Terapia Ocupacional, Faculdade de Medicina, Universidade Federal de Minas Gerais (UFMG), Belo Horizonte (MG), Brasil

Karla Cristhina Alves de Sousa Departamento de Saúde Mental, Faculdade de Medicina, Universidade Federal de Minas Gerais (UFMG), Belo Horizonte (MG), Brasil

Financiamento: Inexistente

Conflito de interesses: Inexistente

Referências

1. Rocha FF, Sousa KCA, Paulino N, Castro JO, Correa H. Suicídio em Belo Horizonte entre 2004 e 2006. Rev Bras Psiquiatr. 2007;29(2): 190-1.

2. Galileu D, Rocha FF, Nicolato R, Teixiera AL, Roamno-Silva MA, Correa $\mathrm{H}$. Brazilian production in psychiatric journals with high impact factor in 2005. J Bras Psiquiatr. 2006;55(2):120-4.

3. Kieling C, Gonçalves RRF. Assessing the quality of a scientific journal: the case of Revista Brasileira de Psiquiatria. Rev Bras Psiquiatr. 2007;29(2):177-81.

4. Razzouk D, Zorzetto R, Dubugras MTB, Gerolin J, Mari JJ. Leading countries in mental health research in Latin America and the Caribbean. Rev Bras Psiquiatr. 2007;29(2):118-22.

5. Botega NJ. Suicídio: saindo da sombra em direção a um Plano Nacional de Prevenção. Rev Bras Psiquiatr. 2007;29(1):7-8. 